\title{
Kinematic and stellar population properties of the counter-rotating components in the S0 galaxy NGC 1366
}

\author{
L. Morelli ${ }^{1,2}$, A. Pizzella ${ }^{1,2}$, L. Coccato ${ }^{3}$, E. M. Corsini ${ }^{1,2}$, E. Dalla Bontà ${ }^{1,2}$, L. M. Buson ${ }^{2}$, V. D. Ivanov ${ }^{3,4}$, \\ I. Pagotto ${ }^{1}$, E. Pompei ${ }^{4}$, and M. Rocco ${ }^{1}$ \\ 1 Dipartimento di Fisica e Astronomia “G. Galilei”, Università di Padova, vicolo dell’Osservatorio 3, 35122 Padova, Italy \\ e-mail: lorenzo.morelli@unipd.it \\ 2 INAF-Osservatorio Astronomico di Padova, vicolo dell'Osservatorio 2, 35122 Padova, Italy \\ 3 European Southern Observatory, Karl-Schwarzschild-Strasse 2, 85748 Garching bei München, Germany \\ ${ }^{4}$ European Southern Observatory, Avenida Alonso de Córdova 3107, Vitacura, 19001 Casilla, Santiago de Chile, Chile
}

Received 11 November 2016 / Accepted 16 January 2017

\begin{abstract}
Context. Many disk galaxies host two extended stellar components that rotate in opposite directions. The analysis of the stellar populations of the counter-rotating components provides constraints on the environmental and internal processes that drive their formation.

Aims. The S0 NGC 1366 in the Fornax cluster is known to host a stellar component that is kinematically decoupled from the main body of the galaxy. Here we successfully separated the two counter-rotating stellar components to independently measure the kinematics and properties of their stellar populations.

Methods. We performed a spectroscopic decomposition of the spectrum obtained along the galaxy major axis and separated the relative contribution of the two counter-rotating stellar components and of the ionized-gas component. We measured the line-strength indices of the two counter-rotating stellar components and modeled each of them with single stellar population models that account for the $\alpha /$ Fe overabundance.

Results. We found that the counter-rotating stellar component is younger, has nearly the same metallicity, and is less $\alpha / \mathrm{Fe}$ enhanced than the corotating component. Unlike most of the counter-rotating galaxies, the ionized gas detected in NGC 1366 is neither associated with the counter-rotating stellar component nor with the main galaxy body. On the contrary, it has a disordered distribution and a disturbed kinematics with multiple velocity components observed along the minor axis of the galaxy.

Conclusions. The different properties of the counter-rotating stellar components and the kinematic peculiarities of the ionized gas suggest that NGC 1366 is at an intermediate stage of the acquisition process, building the counter-rotating components with some gas clouds still falling onto the galaxy.
\end{abstract}

Key words. galaxies: abundances - galaxies: kinematics and dynamics - galaxies: formation - galaxies: stellar content galaxies: individual: 1366

\section{Introduction}

The photometric and kinematic analysis of nearby objects reveals that disk galaxies may host decoupled structures on various scales, from a few tens of pc (e.g., Pizzella et al. 2002; Corsini et al. 2003; Erwin 2004) to several kpc (e.g., Rubin 1994; Kuijken \& Garcia-Ruiz 2001; Combes 2006). In particular, observational evidence for two stellar disks, two gaseous disks, or for a gaseous disk and a stellar disk rotating in opposite directions have been found on large scales in galaxies of different morphological types (Galletta 1996; Corsini 2014). Counter-rotating stellar and/or gaseous disks occur in $\sim 30 \%$ of S0 galaxies (Pizzella et al. 2004; Davis et al. 2011) and in 10\% of spirals (Kannappan \& Fabricant 2001; Pizzella et al. 2004; Corsini et al. 2012).

Different processes have been proposed to explain the formation of a galaxy with two counter-rotating stellar disks, and each formation scenario is expected to leave a noticeable signature in the stellar population properties of the counter-rotating

\footnotetext{
* Based on observations made with ESO Telescopes at the La SillaParanal Observatory under programmes 075.B-0794 and 077.B-0767.
}

components. A counter-rotating stellar disk can be built from gas accreted with an opposite angular momentum with respect to the pre-existing galaxy from the environment or from a companion galaxy. The counter-rotating gas settles on the galaxy disk and forms the counter-rotating stars. In this case, the gas is kinematically associated with the counter-rotating stellar component, which is younger and less massive than the main body of the galaxy (Thakar \& Ryden 1996, 1998; Algorry et al. 2014). Another viable, but less probable, formation process is related to the major merger between two disk galaxies with opposite rotation. The difference in age of the two counter-rotating components depends on the stellar population of the progenitors and on the timescale of the star formation triggered by the binary merger. Moreover, the two stellar disks are expected to have a different thickness (Puerari \& Pfenniger 2001; Crocker et al. 2009; Bettoni et al. 2014). Finally, the dissolution of a bar or triaxial stellar halo can build two counter-rotating stellar components with similar age and mass without involving gas. One of them is rotating in the same direction as the bulge and disk of the pre-existing galaxy (Evans \& Collett 1994, but see also Sellwood \& Merritt 1994; Khoperskov \& Bertin 2017). 
These predictions are difficult to be tested, since outside our Galaxy it is a hard task to separate the single components of a composite stellar population. However, this is possible in a few galaxies because of the difference in velocity of their extended counter-rotating stellar components. Counter-rotating galaxies are therefore ideal laboratories for studying how galaxies grow by episodic or continuous accretion of gas and stars through acquisition and merging events. Coccato et al. (2011) presented a spectroscopic decomposition technique that allows separating the relative contribution of two stellar components from the observed galaxy spectrum. This allows us to study the kinematics and spectroscopic properties of individual components independently, minimizing their cross-contamination along the line of sight. We applied this technique to many of the galaxies known to host counter-rotating stellar disks with the aim of constraining their formation process (Coccato et al. 2011, 2013, 2015; Pizzella et al. 2014). In most of these cases, the available evidence supports the hypothesis that stellar counterrotation is the end product of a retrograde acquisition of external gas and subsequent star formation. Other teams developed their own algorithms for separating the kinematics and stellar populations of counter-rotating galaxies and found results similar to ours (Johnston et al. 2013; Katkov et al. 2011, 2013, 2016; Mitzkus et al. 2017).

NGC 1366 is a bright and spindle galaxy (Fig. 1) in the Fornax cluster at a distance of $17 \mathrm{Mpc}$ (Ferguson 1989). It is classified as $\mathrm{S}^{0}$ by de Vaucouleurs et al. (1991) and $\mathrm{SO}_{1}$ (7)/E7 by Sandage $\&$ Bedke (1994) because it has a highly inclined thin disk. Although NGC 1366 belongs to the LGG 96 group, Garcia et al. (1993), it does not have any nearby bright companion and shows an undisturbed morphology. It has an absolute total $B$ magnitude $M_{B_{T}}^{0}=-18.30 \mathrm{mag}$, as derived from $B_{T}=$ 11.97 mag (de Vaucouleurs et al. 1991) by correcting for the inclination and extinction given by HyperLeda (Makarov et al. 2014). The apparent isophotal diameters measured at a surface brightness level of $\mu_{B}=25 \mathrm{mag} \operatorname{arcsec}^{-2}$ are $2.1 \times 0.9$ arcmin corresponding to $10.4 \times 4.5 \mathrm{kpc}$. Its surface-brightness distribution is well fit by a Sérsic bulge and an exponential disk with a bulge-to-total luminosity ratio $B / T=0.2$, as found by Morelli et al. (2008). These authors detected a kinematically decoupled stellar component that is younger than the host bulge and has probably formed by enriched material acquired through interaction or minor merging.

In this paper we revisit the case of NGC 1366 by successfully separating the two counter-rotating components and properly measuring the properties of their stellar populations (Sect. 2). The analysis of the kinematics of the stars and ionized gas and of the stellar populations is consistent with the formation of the counter-rotating component from external gas that is still accreting onto the galaxy (Sect. 3).

\section{Long-slit spectroscopy}

\subsection{Observations and data reduction}

We carried out the spectroscopic observations of NGC 1366 on 2005 January 25 with the $3.5 \mathrm{~m} \mathrm{New} \mathrm{Technology} \mathrm{Telescope}$ (NTT) at the European Southern Observatory (ESO) in La Silla (Chile). We obtained $2 \times 45$-min spectra along the major $(\mathrm{PA}=$ $\left.2^{\circ}\right)$ and minor $\left(\mathrm{PA}=92^{\circ}\right)$ axis of the galaxy with the ESO MultiMode Instrument (EMMI). It mounted a 1200 grooves $\mathrm{mm}^{-1}$ grating with a $1.0 \operatorname{arcsec} \times 5.5$ arcmin slit, giving an instrumental resolution $\sigma_{\text {inst }}=25 \mathrm{~km} \mathrm{~s}^{-1}$. The detector was a mosaic of the No. 62 and No. 63 MIT/LL CCDs. Each CCD has

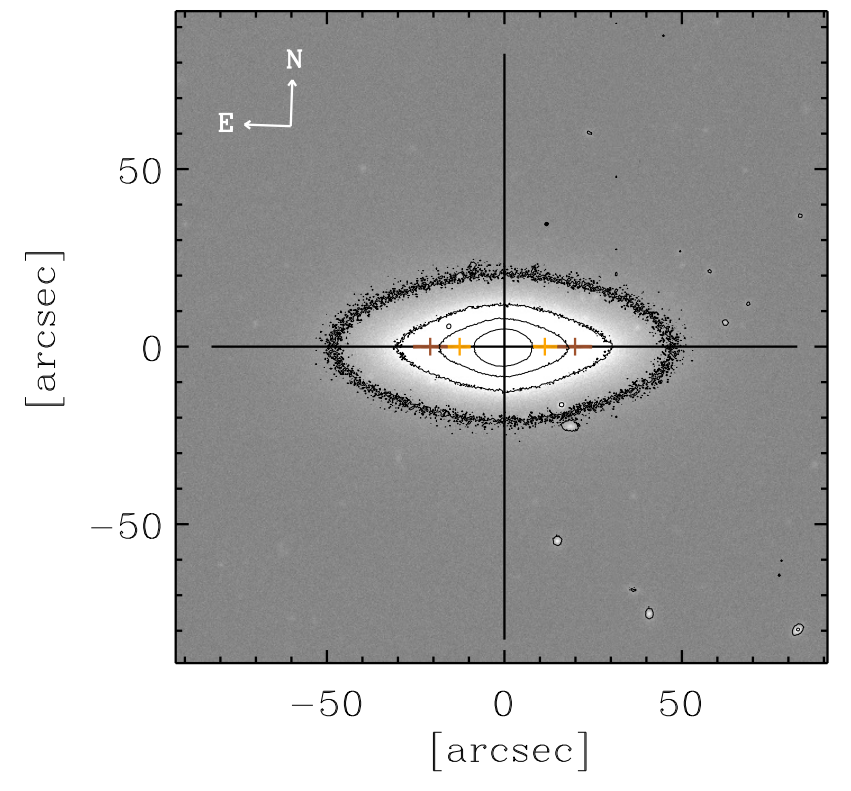

Fig. 1. Contour plots in arbitrary scale of the $R$-band image of NGC 1366 taken from Morelli et al. (2008). The solid lines mark the position of the slit along the major and minor axis of the galaxy. The brown and orange segments correspond to the radial bins where we were able to separate the two counter-rotating stellar components. Orientation of the field of view is given in the figure, and the scale is $82 \mathrm{pc}$ per arcsec.

$2048 \times 4096$ pixels of $15 \times 15 \mu \mathrm{m}^{2}$. We adopted a $2 \times 2$ pixel binning. The wavelength range between about $4800 \AA$ and $5400 \AA$ was covered with a reciprocal dispersion of $0.40 \AA$ pixel $^{-1}$ after $2 \times 2$ pixel binning. All the spectra were bias subtracted, flat-field corrected, cleaned of cosmic rays, and wavelength calibrated using standard IRAF ${ }^{1}$ routines. The spectra obtained along the same axis were coadded using the center of the stellar continuum as reference. Further details about the instrumental setup and spectra acquisition are given in Morelli et al. (2008). We followed the prescriptions of Morelli et al. (2016) for the data reduction.

\subsection{Stellar and ionized-gas kinematics}

We derived the stellar kinematics along both the major and minor axis of NGC 1366 with a single-component and with a twocomponents analysis as done in Pizzella et al. (2014).

We first measured the spectra without separating the two counter-rotating components (Morelli et al. 2015). We used the penalized pixel fitting (pPXF, Cappellari \& Emsellem 2004) and gas and absorption line fitting (GANDALF, Sarzi et al. 2006) IDL $^{2}$ codes with the ELODIE library of stellar spectra from Prugniel \& Soubiran (2001) and adopting a Gaussian line-ofsight velocity distribution (LOSVD) to obtain the velocity curve and velocity dispersion radial profile along the observed axes. We subtracted the measured velocities from the systemic velocity, but we did not apply any correction for the slit orientation and galaxy inclination, while we corrected the measured velocity dispersion for the instrumental velocity dispersion.

1 Image Reduction and Analysis Facility (IRAF) is distributed by the National Optical Astronomy Observatory (NOAO), which is operated by the Association of Universities for Research in Astronomy (AURA), Inc. under cooperative agreement with the National Science Foundation.

2 Interactive Data Language (IDL) is distributed by ITT Visual Information Solutions. 


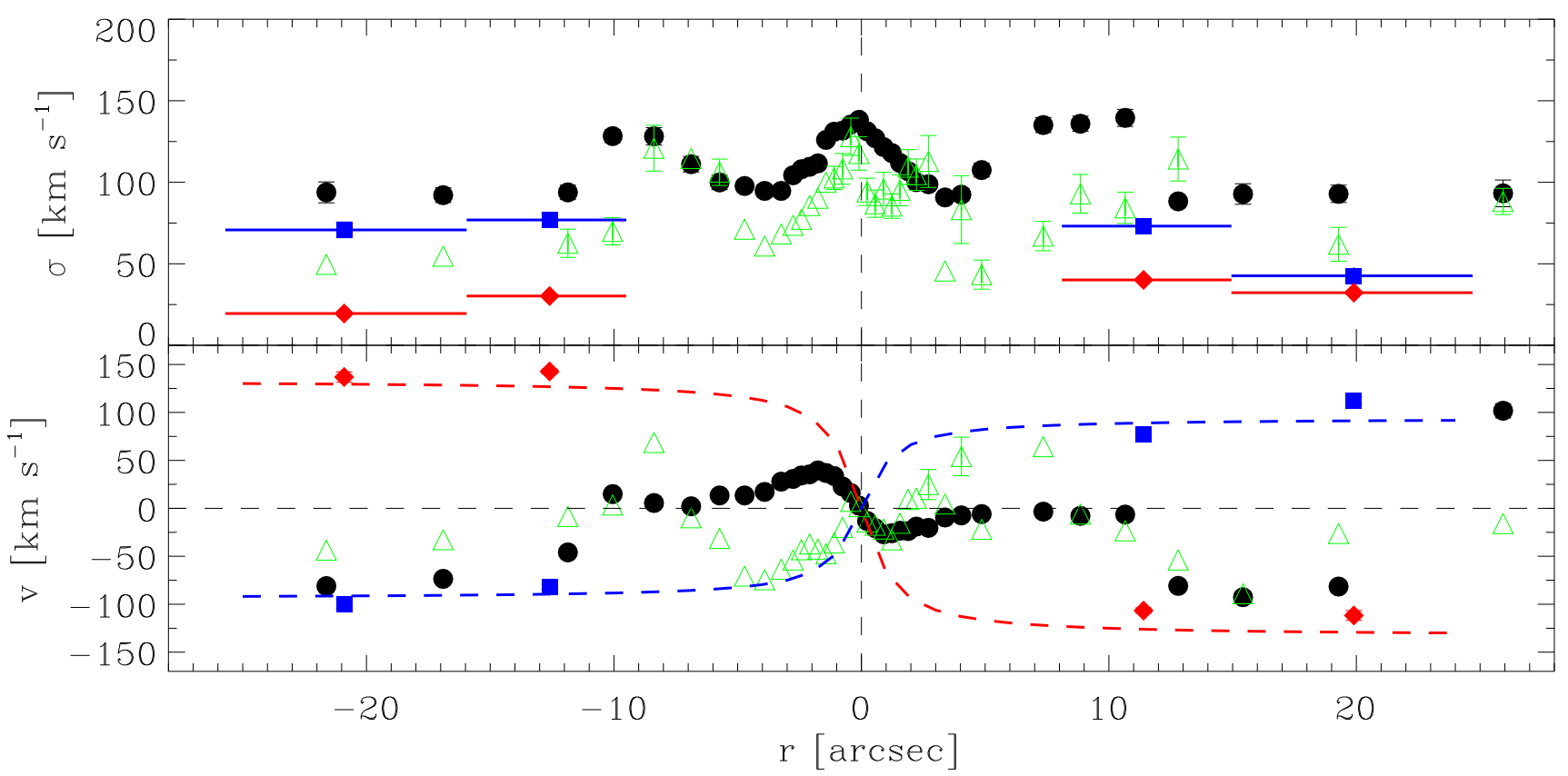

Fig. 2. Line-of-sight velocity dispersion (top panel) and velocity (bottom panel) radial profiles measured along the major axis of NGC 1366 for the total (black filled circles), counter-rotating (blue filled square), and co-rotating (red filled diamonds) stellar components and for the ionized gas component (green open triangles). Error bars smaller than symbols are not shown. The blue and red horizontal lines in the top panel mark the radial bins we adopted for measuring the counter-rotating and corotating components, respectively. The blue and red dashed lines in the bottom panel are a tentative indication of the velocity rotation curves for the counter-rotating and corotating component, respectively.

We found a peculiar stellar kinematics along the major axis of NGC 1366 (Fig. 2). The velocity curve is symmetric around the center for the innermost $|r| \leq 11^{\prime \prime}$. It is characterized by a steep rise reaching a maximum of $|v| \simeq 50 \mathrm{~km} \mathrm{~s}^{-1}$ at $|r| \simeq 2^{\prime \prime}$ and decreasing farther out to $|v| \simeq 0 \mathrm{~km} \mathrm{~s}^{-1}$ at $6 \lesssim|r| \lesssim 11^{\prime \prime}$. For $|r| \geq 11^{\prime \prime}$ the spectral absorption lines clearly display a double peak that is due to the difference in velocity of the two counterrotating components. The absorption lines of the two stellar populations are so well separated that the pPXF-GANDALF procedure fit only one of the two components. This is the reason for the shift in velocities and the drop in velocity dispersion to lower values that we measured on both sides of the galaxy at $|r| \geq 11^{\prime \prime}$ (Fig. 2). The velocities measured at large negative and positive radii are related to the counter-rotating and corotating component, respectively. The velocity dispersion shows a central maximum $\sigma \simeq 150 \mathrm{~km} \mathrm{~s}^{-1}$ and decreases outwards. It rises again to peak at $\sigma \simeq 140 \mathrm{~km} \mathrm{~s}^{-1}$ at $|r| \simeq 9^{\prime \prime}$ and decreases to a value of $\sigma \simeq 100 \mathrm{~km} \mathrm{~s}^{-1}$ at $|r| \simeq 25^{\prime \prime}$. The combination of zero velocity with two off-centered and symmetric peaks in the velocity dispersion of the stellar component measured along the galaxy major axis is indicative of two counter-rotating components. This feature shows up in the kinematics obtained from longslit (Bertola et al. 1996; Vergani et al. 2007) and integral-field spectroscopy (Krajnović et al. 2011; Katkov et al. 2013) when the two counter-rotating components have almost the same luminosity and their difference in velocity is not resolved.

We found no kinematic signature of stellar decoupling along the minor axis of NGC 1366 (Fig. 3). The velocity curve is characterized by $|v| \simeq 0 \mathrm{~km} \mathrm{~s}^{-1}$ at all radii, indicating that the photometric and kinematic minor axes of the galaxy coincide with each other. The velocity dispersion profile is radially symmetric and smoothly declines from $\sigma \simeq 150 \mathrm{~km} \mathrm{~s}^{-1}$ in the center to $\simeq 60 \mathrm{~km} \mathrm{~s}^{-1}$ at the last measured radius $\left(r \simeq 14^{\prime \prime}\right)$.

Finally, we derived the kinematics of the two counterrotating components along the major axis at the radii where their difference in velocity was resolved, giving rise to doublepeaked absorption lines. To reach the signal-to-noise ratio $(\mathrm{S} / \mathrm{N})$ needed to successfully perform the spectral decomposition, we averaged the galaxy spectrum along the spatial direction in the regions with the highest contribution of the counter-rotating component. We obtained a minimum $S / N \geq 30$ per resolution element, which increases to a maximum value $S / N \simeq 50$ in the very central region.

We performed the spectroscopic decomposition using the implementation of the pPXF developed by Coccato et al. (2011). We built for each stellar component a best-fitting synthetic template as linear combination of the ELODIE stellar spectra. The two templates depend on the corresponding stellar populations of the corotating and counter-rotating components and were convolved with a Gaussian LOSVD according to their kinematics. We added multiplicative polynomials to deal with differences in the continuum shape of the galaxy and stellar spectra due to flux calibration and flat fielding residuals. We also included a few Gaussian functions to account for the ionized-gas emission lines and generated a synthetic galaxy spectrum that matches the observed spectrum. The spectroscopic decomposition returns the luminosity fraction, the line-of-sight velocity, and velocity dispersion of the two stellar components, the line-of-sight velocity and velocity dispersion of the ionized gas, and the two bestfitting synthetic stellar templates to be used for the analysis of the stellar population properties. We quantified the errors on the luminosity fraction, line-of-sight velocity, and velocity dispersion of the two counter-rotating stellar components with a series of Monte Carlo simulations on a set of artificial galaxy spectra, as done in Coccato et al. (2011).

The decomposition of the galaxy spectrum in the radial bins at $r=-20.9^{\prime \prime},-12.6^{\prime \prime}, 11.4^{\prime \prime}$ and 19.9" are shown in Fig. 5, and the resulting kinematics of the corotating and counter-rotating stellar components are plotted in Fig. 2. Corotating stars are characterized by a higher rotation velocity $(|v| \simeq 120) \mathrm{km} \mathrm{s}^{-1}$ 


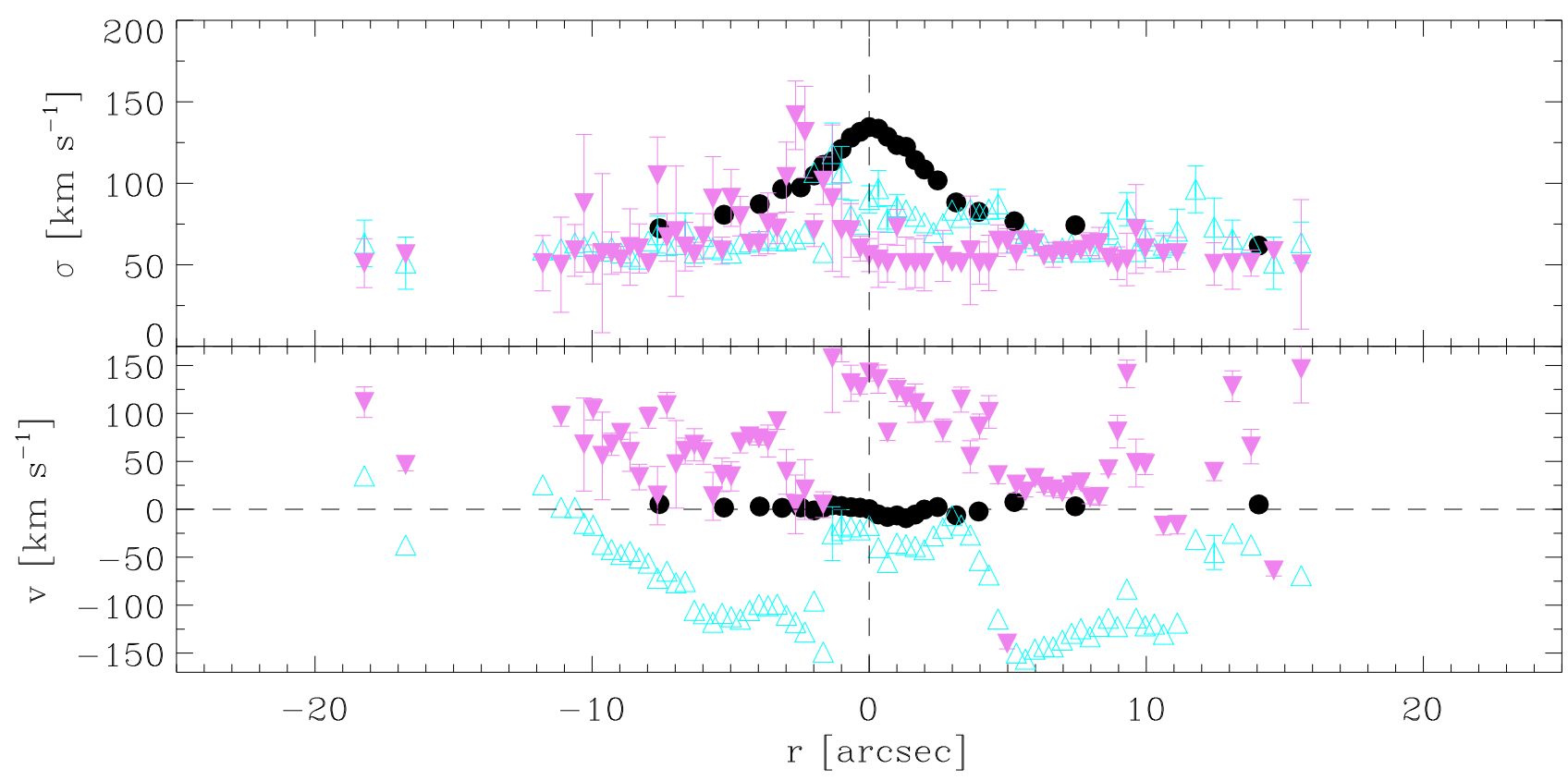

Fig. 3. Line-of-sight velocity dispersion (top panel) and velocity (bottom panel) radial profiles measured along the minor axis of NGC 1366 for the total stellar (black filled circles) and two ionized-gas components (cyan open triangles and violet filled triangles). Error bars smaller than symbols are not shown.

and a lower velocity dispersion $(\sigma \simeq 30)$ than the counterrotating stars that rotate with a $|v| \simeq 90 \mathrm{~km} \mathrm{~s}^{-1}$ and have a $(\sigma \simeq$ $\left.80 \mathrm{~km} \mathrm{~s}^{-1}\right)$. The corotating and counter-rotating components contribute $(45 \pm 15) \%$ and $(55 \pm 15) \%$ of the stellar luminosity at all the measured radii. We converted the luminosity fraction of each component into mass fraction using the measured ages and metallicities and adopting the models by Maraston (2005). We derived stellar mass-to-light ratios of $M / L=3.02$ and $M / L=1.63$ for the corotating and counter-rotating components, respectively. From these quantities we found that the stellar mass fractions of the corotating and counter-rotating components are $60 \%$ and $40 \%$, respectively.

A comparison between the stellar and ionized-gas velocity curves indicates that the gas is disturbed and is not associated with one of the two counter-rotating components. In fact, the gas rotates in the same direction and with a velocity amplitude close to that of the stellar component at small $\left(|r| \lesssim 1^{\prime \prime}\right)$ and large radii $\left(|r| \geq 11^{\prime \prime}\right)$. A broad feature is clearly visible in the gas structure at $|r| \simeq 7-10^{\prime \prime}$ along the major axis (Fig. 4). Although the [O III] $\lambda 5007$ emission line has a broad profile (Fig. 5), there is no clear evidence for a double peak. The wavelength range of our spectra does not cover the $\mathrm{H} \alpha$ region, which prevents us form building a complete diagnostic diagram to properly distinguish between the different excitation mechanisms of the ionized gas. However, the high value of $\log ([\mathrm{O}$ III $] \lambda 5007 / \mathrm{H} \beta) \simeq 1.5$ favors the shocks as excitation mechanism.

We detected two ionized-gas rotating components along the galaxy minor axis as it results from the double-peaked [O III] $\lambda 5007$ emission line shown in Fig. 4. We independently measured the brighter emission line at lower velocities and the fainter emission line at higher velocities. Their velocity and velocity dispersion are shown in Fig. 3. The two gas components have a systematic and almost constant offset in velocity with respect to the stellar component, suggesting the presence of more gas clouds along the line of sight. We prefer this interpretation to the idea of having two gas components with mirrored asymmetric distributions with a brighter and a fainter side and giving rise to an $\mathrm{X}$-shaped [O III] $\lambda 5007$ emission line. The gas velocity dispersion is typically $\sigma_{\text {gas }}<100 \mathrm{~km} \mathrm{~s}^{-1}$ and mostly $\sigma_{\text {gas }} \simeq 50 \mathrm{~km} \mathrm{~s}^{-1}$ along both axes after correcting for the instrumental velocity dispersion.

\subsection{Stellar populations}

We measured the Lick line-strength indices (as defined in Gorgas et al. 1990; Worthey et al. 1994; Thomas et al. 2003) of the corotating and counter-rotating components on the bestfitting synthetic templates and derived the age, metallicity, and $[\alpha / \mathrm{Fe}]$ ratio of the corresponding stellar population as in Morelli et al. (2012). We derived the errors on the equivalent widths of the line-strength indices of the two counter-rotating stellar components with a series of Monte Carlo simulations on a set of artificial galaxy spectra as done in Coccato et al. (2011). We report the measurements in Table 1 and compare them to the line-strength indices predicted for a single stellar population that accounts for the $\alpha / \mathrm{Fe}$ overabundance by Thomas et al. (2003) in Fig. 6. We obtained the stellar population properties of both components from the line-strength indices averaged on the two galaxy sides. They are given in Table 2 together with the relative luminosity of the corotating and counter-rotating components.

The comparison of the averaged age values suggests that the counter-rotating component is significantly younger (Age = $2.6 \mathrm{Gyr}$ ) than the corotating component (age = 5.6 Gyr). The two averaged metallicities are both subsolar and similar to each other $([\mathrm{Z} / \mathrm{H}]=-0.16$ and $-0.18 \mathrm{dex}$ for the counter-rotating and corotating components, respectively). However, the large scatter in the metallicity measurements of the corotating component does not allow us to give a firm conclusion. At face value, the subsolar $[\alpha / \mathrm{Fe}]$ ratio of the counter-rotating component $([\alpha / \mathrm{Fe}]=-0.07 \mathrm{dex})$ points to a longer star-formation timescale than that of the corotating component, which is characterized by a supersolar $[\alpha / \mathrm{Fe}]$ ratio $([\alpha / \mathrm{Fe}]=0.08 \mathrm{dex})$. 

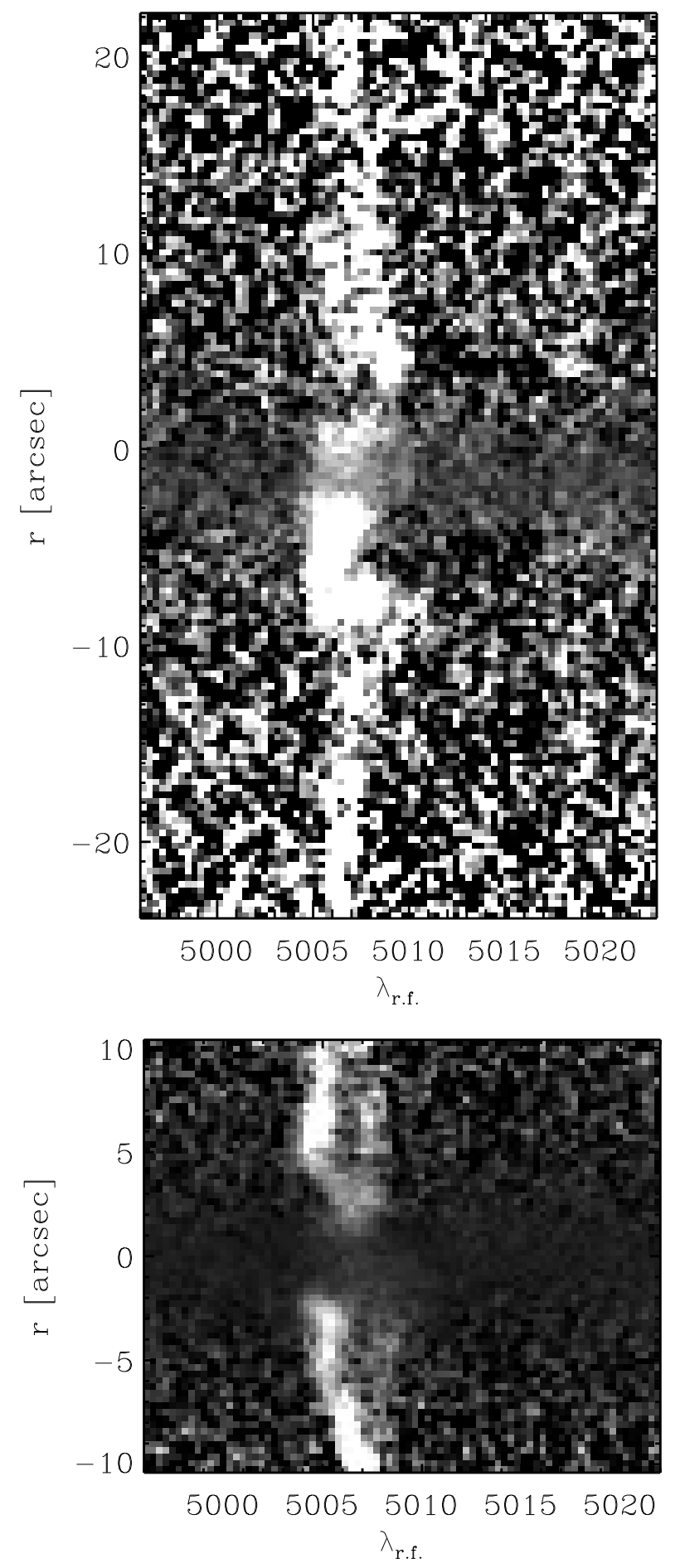

Fig. 4. Portion of major (top panel) and minor-axis (bottom panel) restframe spectra of NGC 1366 showing the [O III] $\lambda 5007$ emission line after subtracting the best-fitting stellar template.

\section{Discussion and conclusions}

There is no morphological or photometric evidence that NGC 1366 is hosting two counter-rotating stellar components. NGC 1366 is characterized by an undisturbed morphology with no sign of recent interaction with small satellites or companion galaxies of similar size (Morelli et al. 2008). This is common for most of the counter-rotating galaxies since their environment does not appear statistically different from that of normal galaxies, see Bettoni et al. (2001). In addition, the surface brightness distribution of NGC 1366 is remarkably well fitted by a Sérsic bulge and an exponential disk with no break at any radius (Morelli et al. 2008).

We provided the spectroscopic evidence of two counterrotating stellar components with a high rotation velocity and low velocity dispersion $(v / \sigma \simeq 2)$ that give almost the same contribution to the galaxy luminosity. We infer that they have a similar scale length from the constant slope of the exponential surface-brightness radial profile outside the bulge-dominated region as in NGC 4138 (Jore et al. 1996; Pizzella et al. 2014) and NGC 4550 (Rix et al. 1992; Coccato et al. 2013; Johnston et al. 2013). These kinematic and photometric properties support the disk nature of the two components.

The stellar population of the corotating component is characterized by an older age, consistent with that of bulge $(5.1 \pm$ 1.7 Gyr, Morelli et al. 2008), subsolar metallicity, and almost so$\operatorname{lar} \alpha / \mathrm{Fe}$ enhancement. This suggests a formation timescale of a few Gyr that occurred at the time of the galaxy assembly. The counter-rotating stellar component is remarkably younger with lower $\alpha / \mathrm{Fe}$ enhancement and subsolar metallicity. The metallicity and age values obtained for the two components are consistent within the errors with the results obtained by Morelli et al. (2008) on the galaxy integrated light when considering its strong radial gradients of stellar population properties. Therefore, the counter-rotating stellar component could be the end result of a slower star formation process that occurred in a disk of gas accreted by a preexisting galaxy and settled onto retrograde orbits. However, unlike most of previously studied cases (e.g., Johnston et al. 2013; Pizzella et al. 2014; Coccato et al. 2015; Katkov et al. 2016), the ionized gas of NGC 1366 is not associated with the counter-rotating stellar component. It has peculiar kinematics with multiple velocity components along the minor axis with different gas clouds along the line of sight. The kinematic mismatch between the ionized gas and counter-rotating stellar component complicates the scenario of gas accretion followed by star formation.

The most obvious possibility is to consider an episodic gas accretion. The first event of capture of external gas occurred $\sim 3$ Gyr ago and built the counter-rotating stellar component. It was followed by a subsequent event that is still ongoing at present. However, this rises the question about the origin of the newly supplied and kinematically decoupled gas since there is no clear donor candidate in the neighborhood of NGC 1366. This leaves us with the possibility of the acquisition of small gas clouds coming either from the environment or from the internal reservoir inside the galaxy itself. When external gas is captured in distinct clouds, it settles onto the galaxy disk in a relatively short time ( $\sim 1 \mathrm{Gyr}$, Thakar et al. 1997; Algorry et al. 2014; Mapelli et al. 2015). In this case, NGC 1366 could be an object caught at an intermediate stage of the acquisition process, before its configuration becomes stable. It is interesting to note that this could also have occurred in galaxies with gas associated with the counter-rotating stellar component. Without clear evidence of ongoing star formation or very young stars, the counter-rotating stellar component could be the result of a past acquisition of gas coming from the same reservoir that provides the counter-rotating gas we observe at present.

An intriguing alternative was explored by Crocker et al. (2009). They showed the time evolution of the distribution and kinematics of gas and stars in a set of numerical simulations aimed at investigating the formation of the stellar counterrotating disks of NGC 4550 from a binary merger. One Gyr after the merger, while the stars have settled in two counter-rotating disks with a relatively regular kinematics, the gas distribution 


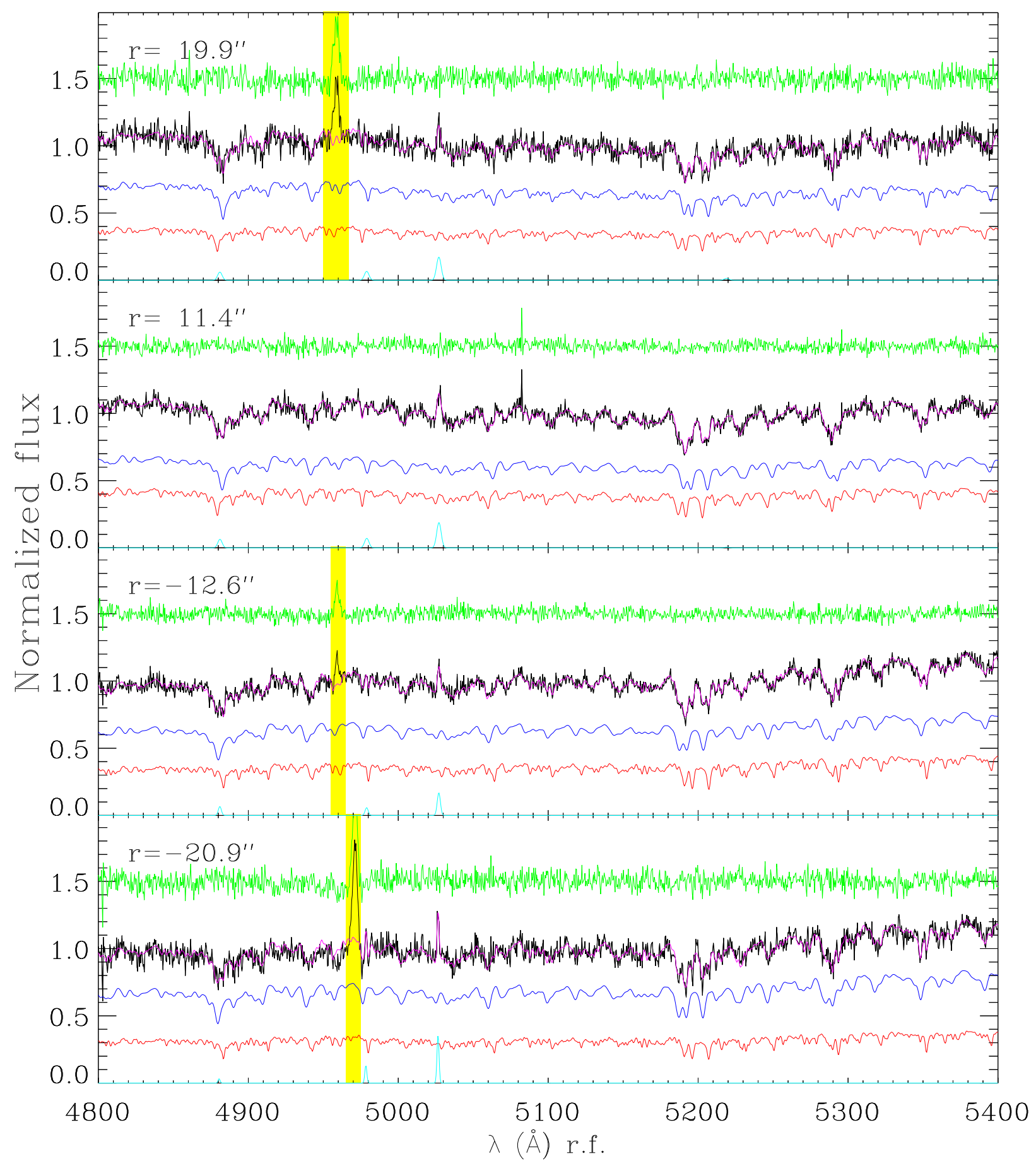

Fig. 5. Decomposition of the major-axis spectrum of NGC 1366 (black line) in the analyzed spatial bins $\left(r=-20.9^{\prime \prime},-12.6^{\prime \prime}, 11.4^{\prime \prime}\right.$ and $\left.19.9^{\prime \prime}\right)$. The best-fitting model (magenta line) is the sum of the spectra of the corotating (red line) and counter-rotating stellar component (blue line) and of the ionized-gas component (cyan line). The normalized flux of the fit residual (green line) has a false zero-point for viewing convenience. The yellow shaded area indicates a spectral region masked in the fit that is due to the imperfect subtraction of the spurious signal, which is the result of a reflection on the EMMI CCD.

still remains rather disordered with a disturbed kinematics. However, this configuration is not stable, and the gas tends to a more regular configuration between 1 and 2 Gyr from the merging event. The structure and stellar populations properties of the counter-rotating components of NGC 1366 are somewhat different from those of NGC 4550 for a direct comparison of our results with the simulations by Crocker et al. (2009), and dedicated simulations are needed for a firmer interpretation of this galaxy in terms of a binary merger.
These speculations need further evidence since the available spectroscopic data are not conclusive. To date, NGC 1366 is a unique example, and it may become a corner stone for understanding the formation of counter-rotation in relatively isolated and undisturbed galaxies. Mapping the ionized-gas distribution and kinematics of NGC 1366 with integral-field spectroscopy is a crucial complement for the present dataset and is necessary to distinguish between different scenarios and address the question of the origin of the gas. In the case of a episodic gas acquisition, 

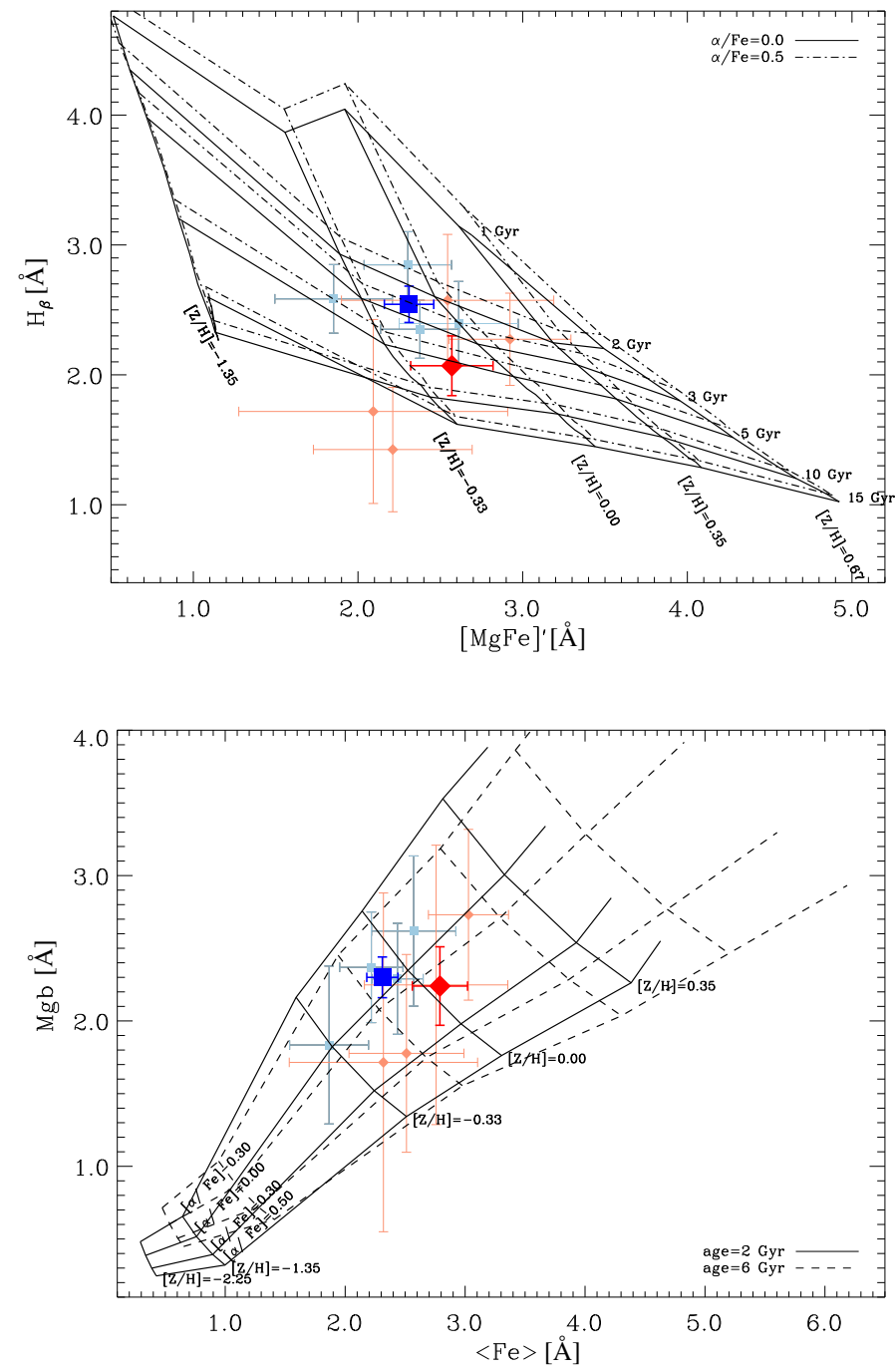

Fig. 6. Values of $\mathrm{H} \beta$ and $[\mathrm{MgFe}]^{\prime}$ line-strength indices (top panel) and $\langle\mathrm{Fe}\rangle$ and $\mathrm{Mg}_{b}$ line-strength indices (bottom panel) for the corotating (small red diamonds) and counter-rotating stellar component (small blue squares) measured along the major axis of NGC 1366 $\left(r=-20.9^{\prime \prime},-12.6^{\prime \prime}, 11.4^{\prime \prime}\right.$, and 19.9"). The larger symbols correspond to the averaged line-strength indices for the two stellar components. The lines indicate the model predictions by Thomas et al. (2003) for different $[\alpha / \mathrm{Fe}]$ ratios (top panel) and ages (bottom panel).

Table 1. Line-strength indices of the corotating and counter-rotating stellar components of NGC 1366.

\begin{tabular}{|c|c|c|c|c|}
\hline $\begin{array}{c}r \\
{\left[{ }^{\prime \prime}\right]}\end{array}$ & $\begin{array}{l}\mathrm{H} \beta \\
{[\AA]}\end{array}$ & $\begin{array}{c}\mathrm{Mg}_{b} \\
{[\AA ̊]}\end{array}$ & $\begin{array}{c}\mathrm{Fe}_{5270} \\
{[\AA ̊}\end{array}$ & $\begin{array}{c}\mathrm{Fe}_{5335} \\
{[\AA]}\end{array}$ \\
\hline \multicolumn{5}{|c|}{ Corotating component } \\
\hline-20.9 & $1.72 \pm 0.71$ & $2.32 \pm 0.79$ & $2.11 \pm 0.89$ & $1.32 \pm 0.80$ \\
\hline-12.6 & $1.43 \pm 0.48$ & $2.51 \pm 0.48$ & $2.16 \pm 0.47$ & $1.39 \pm 0.49$ \\
\hline 11.4 & $2.27 \pm 0.36$ & $3.03 \pm 0.33$ & $2.94 \pm 0.39$ & $2.52 \pm 0.44$ \\
\hline 19.9 & $2.57 \pm 0.51$ & $2.76 \pm 0.60$ & $2.47 \pm 0.68$ & $2.02 \pm 0.68$ \\
\hline \multicolumn{5}{|c|}{ Counter-rotating component } \\
\hline-20.9 & $2.40 \pm 0.32$ & $2.57 \pm 0.32$ & $2.70 \pm 0.38$ & $2.54 \pm 0.35$ \\
\hline-12.6 & $2.85 \pm 0.26$ & $2.22 \pm 0.26$ & $2.42 \pm 0.27$ & $2.32 \pm 0.27$ \\
\hline 11.4 & $2.35 \pm 0.22$ & $2.44 \pm 0.21$ & $2.35 \pm 0.25$ & $2.23 \pm 0.29$ \\
\hline 19.9 & $2.59 \pm 0.26$ & $1.87 \pm 0.33$ & $1.84 \pm 0.39$ & $1.83 \pm 0.38$ \\
\hline
\end{tabular}

Table 2. Properties of the stellar populations of the corotating and counter-rotating stellar components of NGC 1366.

\begin{tabular}{lcccc}
\hline \hline \multicolumn{1}{c}{ Component } & $L / L_{\mathrm{T}}$ & Age & {$[\mathrm{Z} / \mathrm{H}]$} & {$[\alpha / \mathrm{Fe}]$} \\
& & {$[\mathrm{Gyr}]$} & {$[\mathrm{dex}]$} & {$[\mathrm{dex}]$} \\
\hline Corotating & 0.45 & $5.6 \pm 2.7$ & $-0.18 \pm 0.16$ & $0.08 \pm 0.13$ \\
Counter-rotating & 0.55 & $2.6 \pm 0.5$ & $-0.16 \pm 0.11$ & $-0.07 \pm 0.08$ \\
\hline
\end{tabular}

we expect to see a clear morphological and kinematic signature of the incoming gas without a counter-part in the stellar distribution. In contrast, in the case of a galaxy binary merger, we expect to observe a morphological association between the distribution of stars and gas, a regular velocity field for the two counter-rotating stellar disks, and an irregular velocity field for the ionized gas.

Acknowledgements. We benefited from discussion with Roberto P. Saglia. This work was supported by Padua University through grants 60A02-5857/13, 60A02-5833/14, 60A02-4434/15, and CPDA133894. L.M. and E.M.C. acknowledge financial support from Padua University grants CPS0204 and BIRD164402/16, respectively. L.M. is grateful to the ESO Scientific Visitor Programme for the hospitality at ESO Headquarters while this paper was in progress. This research made use of the HyperLeda Database (http://leda. univ-lyon 1.fr/) and NASA/IPAC Extragalactic Database (NED) which is operated by the Jet Propulsion Laboratory, California Institute of Technology, under contract with the National Aeronautics and Space Administration (http://ned.ipac. caltech.edu/).

\section{References}

Algorry, D. G., Navarro, J. F., Abadi, M. G., et al. 2014, MNRAS, 437, 3596 Bertola, F., Cinzano, P., Corsini, E. M., et al. 1996, ApJ, 458, L67 Bettoni, D., Galletta, G., \& Prada, F. 2001, A\&A, 374, 83

Bettoni, D., Mazzei, P., Rampazzo, R., et al. 2014, Ap\&SS, 354, 83 Cappellari, M., \& Emsellem, E. 2004, PASP, 116, 138

Coccato, L., Morelli, L., Corsini, E. M., et al. 2011, MNRAS, 412, L113

Coccato, L., Morelli, L., Pizzella, A., et al. 2013, A\&A, 549, A3

Coccato, L., Fabricius, M., Morelli, L., et al. 2015, A\&A, 581, A65

Combes, F. 2006, in Mass Profiles and Shapes of Cosmological Structures, eds. G. A. Mamon, F. Combes, C. Deffayet, \& B. Fort, EAS Pub. Ser., 20, 97

Corsini, E. M. 2014, in Multi-Spin Galaxies, eds. E. Iodice, \& E. M. Corsini, ASP Conf. Ser., 486, 51

Corsini, E. M., Pizzella, A., Coccato, L., \& Bertola, F. 2003, A\&A, 408, 873 Corsini, E. M., Méndez-Abreu, J., Pastorello, N., et al. 2012, MNRAS, 423, L79 Crocker, A. F., Jeong, H., Komugi, S., et al. 2009, MNRAS, 393, 1255 Davis, T. A., Alatalo, K., Sarzi, M., et al. 2011, MNRAS, 417, 882 de Vaucouleurs, G., de Vaucouleurs, A., Corwin, H. G., et al. 1991, Third Reference Catalogue of Bright Galaxies (Berlin: Springer)

Erwin, P. 2004, A\&A, 415, 941

Evans, N. W. \& Collett, J. L. 1994, ApJ, 420, L67

Ferguson, H. C. 1989, AJ, 98, 367

Galletta, G. 1996, in Barred Galaxies, eds. R. Buta, D. A. Crocker, \& B. G. Elmegreen, ASP Conf. Ser., 91, 429

Garcia, A. M., Paturel, G., Bottinelli, L., \& Gouguenheim, L. 1993, A\&AS, 98, 7

Gorgas, J., Efstathiou, G., \& Aragón-Salamanca, A. 1990, MNRAS, 245, 217

Johnston, E. J., Merrifield, M. R., Aragón-Salamanca, A., \& Cappellari, M. 2013, MNRAS, 428, 1296

Jore, K. P., Broeils, A. H., \& Haynes, M. P. 1996, AJ, 112, 438

Kannappan, S. J., \& Fabricant, D. G. 2001, AJ, 121, 140

Katkov, I., Chilingarian, I., Sil'chenko, O., Zasov, A., \& Afanasiev, V. 2011, Balt. Astron., 20, 453

Katkov, I. Y., Sil'chenko, O. K., \& Afanasiev, V. L. 2013, ApJ, 769, 105

Katkov, I. Y., Sil'chenko, O. K., Chilingarian, I. V., Uklein, R. I., \& Egorov, O. V. 2016, MNRAS, 461, 2068

Khoperskov, S., \& Bertin, G. 2017, A\&A, 597, A103

Krajnović, D., Emsellem, E., Cappellari, M., et al. 2011, MNRAS, 414, 2923

Kuijken, K., \& Garcia-Ruiz, I. 2001, in Galaxy Disks and Disk Galaxies, eds. J. G. Funes, \& E. M. Corsini, ASP Conf. Ser., 230, 401 
Makarov, D., Prugniel, P., Terekhova, N., Courtois, H., \& Vauglin, I. 2014, A\&A 570, A13

Mapelli, M., Rampazzo, R., \& Marino, A. 2015, A\&A, 575, A16

Maraston, C. 2005, MNRAS, 362, 799

Mitzkus, M., Cappellari, M., \& Walcher, C. J. 2017, MNRAS, 464, 4789

Morelli, L., Pompei, E., Pizzella, A., et al. 2008, MNRAS, 389, 341

Morelli, L., Corsini, E. M., Pizzella, A., et al. 2012, MNRAS, 423, 962

Morelli, L., Pizzella, A., Corsini, E. M., et al. 2015, Astron. Nachr., 336, 208

Morelli, L., Parmiggiani, M., Corsini, E. M., et al. 2016, MNRAS, 463, 4396

Pizzella, A., Corsini, E. M., Morelli, L., et al. 2002, ApJ, 573, 131

Pizzella, A., Corsini, E. M., Vega Beltrán, J. C., \& Bertola, F. 2004, A\&A, 424 447

Pizzella, A., Morelli, L., Corsini, E. M., et al. 2014, A\&A, 570, A79

Prugniel, P., \& Soubiran, C. 2001, A\&A, 369, 1048
Puerari, I., \& Pfenniger, D. 2001, Ap\&SS, 276, 909

Rix, H.-W., Franx, M., Fisher, D., \& Illingworth, G. 1992, ApJ, 400, L5 Rubin, V. C. 1994, AJ, 108, 456

Sandage, A., \& Bedke, J. 1994, The Carnegie Atlas of Galaxies (Washington, DC: Carnegie Institution of Washington)

Sarzi, M., Falcón-Barroso, J., Davies, R. L., et al. 2006, MNRAS, 366, 1151

Sellwood, J. A., \& Merritt, D. 1994, ApJ, 425, 530

Thakar, A. R., \& Ryden, B. S. 1996, ApJ, 461, 55

Thakar, A. R., \& Ryden, B. S. 1998, ApJ, 506, 93

Thakar, A. R., Ryden, B. S., Jore, K. P., \& Broeils, A. H. 1997, ApJ, 479, 702

Thomas, D., Maraston, C., \& Bender, R. 2003, MNRAS, 339, 897

Vergani, D., Pizzella, A., Corsini, E. M., et al. 2007, A\&A, 463, 883

Worthey, G., Faber, S. M., Gonzalez, J. J., \& Burstein, D. 1994, ApJS, 94, 687 